\title{
Microscopy-based Methods for Rosetting Assay in Malaria Research
}

Wenn-Chyau Lee* and Laurent Rénia*

Singapore Immunology Network (SIgN), Agency for Science, Technology and Research (A*STAR), Singapore

*For correspondence: leewc 88@hotmail.com; renia laurent@immunol.a-star.edu.sg

\begin{abstract}
[Abstract] In malaria, rosetting phenomenon is a condition where a Plasmodium-infected erythrocyte stably adheres to at least an uninfected erythrocyte. This phenomenon that occurs in all species of human malaria parasite is likely to be an immune escape mechanism for the parasite. However, it has been associated with malaria pathogenesis, possibly by facilitating microvasculature occlusion along with direct endothelial cytoadherence by the infected erythrocytes. There are different microscopy-based techniques to visualize rosettes but neither of these techniques has yet to qualify as the official "gold standard" method. We have found that these techniques can be used interchangeably, provided that the conditions of the experiments are properly controlled. Here, we presented three methods as options for rosetting assay, i.e., the unstained wet mount technique, acridine orange based-fluorescence microscopy technique and Giemsa stained wet mount method, with preparation steps that enable consistent performance in rosetting experiments.
\end{abstract}

Keywords: Malaria, Rosettes, Wet mount, Microscope, Live intracellular parasite staining

[Background] Malaria remains a serious healthcare problem in many countries across the tropical and sub-tropical regions (World Health Organization, 2019). As the parasite matures within the host's erythrocyte, substantial changes happen to the architecture of the infected erythrocyte, giving rise to altered cytoadherence of the infected erythrocytes, which is believed to be an important player in pathogenesis of severe malaria (Craig et al., 2012). One of the cytoadherence characteristics of infected erythrocyte is the rosetting phenomenon, where a late-stage parasite-infected erythrocyte stably adheres to at least an uninfected erythrocyte (Figure 1). Since its discovery in the late 1980s (David et al., 1988), various methods have been used to visualize rosettes. Most of these methods are microscopy-based, using wet mount prepared with malaria-infected blood samples. These include unstained wet mount (Udomsangpetch et al., 1992), Giemsa-stained wet mount (Chotivanich et al., 1998; Lee et al., 2013 and 2014), and fluorescent dye Acridine Orange-stained wet mount (Udomsangpetch et al., 1989; Treutiger et al., 1998; Ribacke et al., 2013). These methods were easy to use and can be conducted even in less-equipped laboratories. Here, we have provided protocols for the three methods, along with the steps of sample preparation for these techniques. When done properly, these methods can be used interchangeably according to the resources available in the work place. 


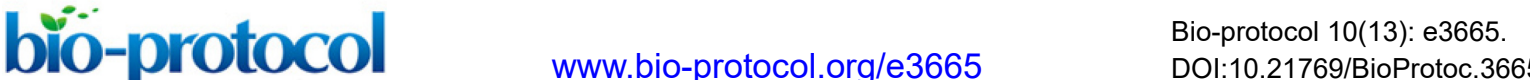

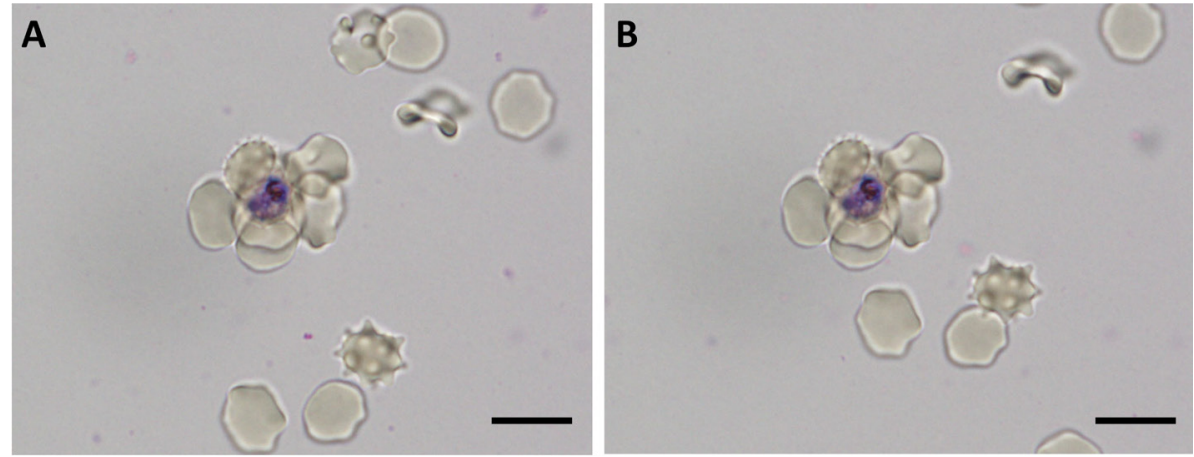

Figure 1. Pictures of a rosette taken 15s apart, Giemsa stained-wet mount method. Under wet mount condition, the cells are mobile, which helps to differentiate between rosettes and mere overlaps of cells. The Giemsa stain assists in visualizing the parasites. Scale bars: $10 \mu \mathrm{m}$.

\section{Materials and Reagents}

1. Cellulose acetate syringe filter, pore size $0.45 \mu \mathrm{m}$ (Sartorius Minsart ${ }^{\circledR}$, Sigma-Aldrich, catalog number: 16555-K)

2. Falcon ${ }^{\circledR}$ cell culture flask T25, filter cap (VWR ${ }^{\mathrm{TM}}$, catalog number: 29185298$)$

3. Glass coverslip $22 \times 32 \mathrm{~mm}$ (Mariendfeld, catalog number: 0101112)

4. Glass slide (Sail Brand, catalog number: 7105)

5. Erythrocytes (from clinical isolates or from parasite cultures)

6. Acridine Orange (Thermo Fisher Scientific, catalog number: 1301)

7. Anti-A (ABO1) murine monoclonal antibody for blood $A B O$ group identification (TransClone ${ }^{\circledR}$ Bio-Rad, catalog number: 86328)

8. Anti-B (ABO1) murine monoclonal antibody for blood $A B O$ group identification (TransClone ${ }^{\circledR}$ Bio-Rad, catalog number: 86470)

9. $\quad$ BD Vacutainer ${ }^{\mathrm{TM}}$ with lithium heparin (Thermo Fisher Scientific, catalog number: 02-657-28)

10. Giemsa (Merck, catalog number: HX60416604)

11. RPMI 1640 medium (HyClone ${ }^{\mathrm{TM}}$, catalog number: $\left.\mathrm{SH} 30255.01\right)$

12. Serum (Human, blood group type-matching or $A B$ group)

13. Giemsa stock preparation (see Recipes)

14. Media (see Recipes)

\section{Equipment}

1. Epifluorescence microscope (Nikon Eclipse, model: TS100)

2. Light microscope (Olympus, model: BX43)

3. Hand tally counter 


\section{Procedure}

1. Plasmodium falciparum parasites were cultured using RPMI 1640 medium enriched with at $20 \%$ blood group type-matching serum (For P. vivax, McCoy's 5A medium can be used).

2. When majority of the parasite population has reached late stages, the culture suspension can be used for experiments.

3. Prepare an aliquot of the culture suspension, and process with either of the three methods (Steps 4-6), according to the feasibility of the techniques in the laboratory.

4. Stain the suspension with Giemsa ( $5 \%$ working concentration volume by volume) for $15 \mathrm{~min}$ prior to wet mount preparation for rosetting assay.

5. Alternatively, add Acridine Orange (working concentration $2 \mu \mathrm{g} / \mathrm{ml}$ ) into the suspension and allow staining for $15 \mathrm{~min}$ prior to wet mount preparation for rosetting.

6. The third method is to use the suspension for wet mount preparation without staining.

7. Wet mount is prepared by pipetting small volume of the suspension above onto a clean glass slide, and cover the droplet with a clean glass coverslip by landing the coverslip slowly to minimize trapment of air bubbles.

8. The Giemsa-stained and unstained wet mount can be viewed with light microscope (of any model), whereas the Acridine Orange-stained wet mount can only be viewed with fluorescence microscope (of any model).

9. Determine the rosetting rates by recruiting 200 infected erythrocytes. Rosetting rate is the percentage of infected erythrocytes that adhere to uninfected erythrocytes.

\section{Data analysis}

Detailed information of data analyses are available in research article (Lee et al., 2020), where the rosetting rates of $P$. falciparum were collected using the three rosetting assay methods described above, and no significant difference were found among the methods (Supplementary file 7. Method comparison for rosetting assay).

\section{Notes}

1. Serum is needed to allow rosette formation.

2. It is important to type-match the ABO blood group of the erythrocytes used and the sera used for enrichment of culture media. This is to avoid agglutination of the cells (Sazama, 1990; Williamson et al., 1999). If the $A B O$ blood group cannot be determined, use only $A B$ serum.

3. Laboratory adapted parasite lines and clinical isolates can be used. For clinical isolates, blood should be collected with lithium heparin-vacutainer, and the cells should be washed at least twice with RPMI 1640 medium prior to use in culture or experiment to remove the anti-coagulant. 
4. Try to avoid using EDTA-vacutainer to collect blood samples for rosetting assay. All anticoagulants hamper rosette formation. However, we noticed that the rosette-inhibitory effect by EDTA is more difficult to be restored after removal of the anticoagulant, which is similar to an earlier report on another chelating agent, EGTA (Chotivanich et al., 1998).

5. Staging of the parasite is determined by reading the Giemsa/ Field-stained blood smear with light microscope. Late stages/mature stages are parasite blood forms with visible malaria pigment (yellowish brown-black pigments) accumulation within the parasite. The expression of rosetting ligands on the surface of infected erythrocytes is optimal at the late stages (Lee et al., 2019).

6. Hematocrit of the suspension should be between $1.5 \%$ and $4 \%$. Suspension with very low hematocrit (Figure 2A) will slow down the wet mount reading process, and cells tend to move a lot under such condition, hampering smooth counting process of rosetting rate. Suspension with very high hematocrit (Figure $2 \mathrm{~B}$ ) will result in "crowded cell" condition, which hampers confident differentiation of rosettes from mere overlaps of cells.
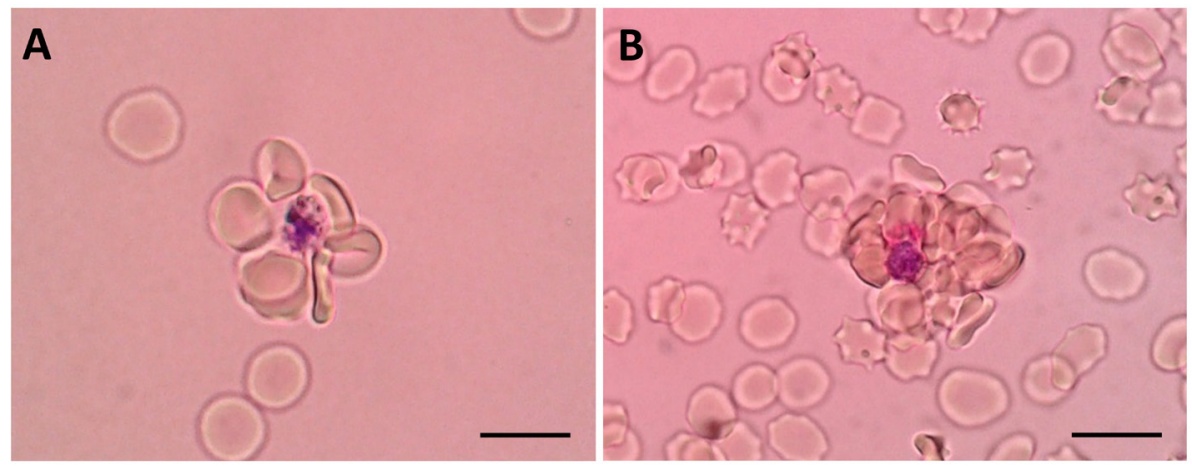

Figure 2. Rosettes found in cell suspension of $1 \%$ hematocrit $(A)$ and $5 \%$ hematocrit (B). Rosettes can still be visualized. However, it may affect the speed or accuracy of the rosette characterization work. Scale bars: $10 \mu \mathrm{m}$.

7. Giemsa allows clear visualization of the parasites. Under wet mount, Giemsa allows differentiation of normocytes (matured erythrocytes) and reticulocytes (young/immature erythrocytes), which may be an important hematological parameter for certain experiments.

8. The unstained wet mount is the simplest way forward for rosetting assay. However, one will need to adjust the light intensity and play with the contrast enhancing features of the microscope to allow clear visualization of the unstained parasites.

9. Resuspend the cell suspension prior to wet mount preparation to ensure the correct hematocrit of cell suspension.

10. The volume of cell suspension for wet mount-making depends on the size of the glass coverslip used. If the volume is too small for the coverslip, the suspension will be "smeared" and "look dried", which become unreadable for rosetting assay. As the wet mount is examined under 
strong light of microscopy, evaporation happens. With small volume, evaporation is fast, creating more hyperosmotic condition in the extracellular environment, which renders the cells crenated.

11. If the volume is too big for a small coverslip, the cells will "move" rapidly and it hampers the examination.

12. For glass coverslip of size $22 \times 32 \mathrm{~mm}$, volume of 7-8 $\mu \mathrm{l}$ is suitable.

13. Never read a wet mount for too long. Crenation of cells starts to happen minutes after the wet mount is placed on the microscope stage, lit by strong bright light (due to evaporation). The cells around the rim/edge of the glass coverslips are affected first, and the effect will spread inward. Once the cells are crenated, the wet mount is no longer suitable for rosetting assay and new wet mount should be prepared.

14. Clean glass slides (oil/grease-free) should be used for the wet mount preparation.

15. Rosetting is a phenomenon that is seen in the late stages. If the parasite suspension used is not stage-synchronised, the worker should not include the ring stages into their rosetting rate counting.

\section{$\underline{\text { Recipes }}$}

1. Giemsa stock preparation

a. Filter the dye stock solution with $0.45 \mu \mathrm{m}$ filter before use. This is to ensure clear and clean visualization of parasites in wet mount.

b. For the dye intended for wet mount staining, the dye filtering should be done once per month.

c. Alternately, filter paper can be used for the filtration. Nevertheless, do not filter a large volume of dye and keep it for too long.

d. The stock should not be exposed to direct sun light.

2. Media

Enrich the plain medium with $20 \%$ type-matching heat-inactivated human serum. For instance, $10 \mathrm{ml}$ of human serum is added to $40 \mathrm{ml}$ of plain medium to prepare $50 \mathrm{ml}$ of medium needed. RPMI1640 medium is used. However, McCoy's 5A medium that is used for $P$. vivax maturation assay can be used for rosetting assays on $P$. vivax as well.

Notes:

a. Fresh media should be used for the experiment.

b. Pay attention to the color of the media. Old media that are usually more intense pinkish (alkaline) may affect the staining efficiency of the dyes.

\section{Acknowledgments}

LR was supported by core funding from $A^{*}$ STAR to SIgN. LR were also funded by $A^{*}$ STAR grant (JCO-DP BMSI/15-800006-SIGN). WCL was funded by Open Fund- Young Individual Research Grant (OF-YIRG NMRC/OFYIRG/0070/2018) by the National Medical Research Council, Ministry of 
Please cite this article as: Lee and Rénia, (2020). Microscopy-based Methods for Rosetting Assay in Malaria Research,Bio-protocol 10 (13): e3665. DOI:

Health, Singapore. This protocol is derived from published work: Lee et al., 2020.

\section{Competing interests}

The authors have no competing interest to declare.

\section{Ethics}

Malaria-infected samples were collected in Shoklo Malaria Research Unit (SMRU) under approved ethics: OXTREC 04-10 (University of Oxford, UK); TMEC 09-082 (Ethics Committee, Faculty of Tropical Medicine, Mahidol University, Thailand).

\section{$\underline{\text { References }}$}

1. Chotivanich, K. T., Pukrittayakamee, S., Simpson, J. A., White, N. J. and Udomsangpetch, R. (1998). Characteristics of Plasmodium vivax-infected erythrocyte rosettes. Am J Trop Med Hyg 59(1): 73-76.

2. Craig, A. G., Khairul, M. F. and Patil, P. R. (2012). Cytoadherence and severe malaria. Malays $J$ Med Sci 19(2): 5-18.

3. David, P. H., Handunnetti, S. M., Leech, J. H., Gamage, P. and Mendis, K. N. (1988). Rosetting: a new cytoadherence property of malaria-infected erythrocytes. Am J Trop Med Hyg 38(2): 289297.

4. Lee, W. C., Malleret, B., Lau, Y. L., Mauduit, M., Fong, M. Y., Cho, J. S., Suwanarusk, R., Zhang, R., Albrecht, L., Costa, F. T., Preiser, P., McGready, R., Renia, L., Nosten, F. and Russell, B. (2014). Glycophorin C (CD236R) mediates vivax malaria parasite rosetting to normocytes. Blood 123(18): e100-109.

5. Lee, W. C., Russell, B. and Renia, L. (2019). Sticking for a Cause: The Falciparum Malaria Parasites Cytoadherence Paradigm. Front Immunol 10: 1444.

6. Lee, W. C., Russell, B., Lau, Y. L., Fong, M. Y., Chu, C., Sriprawat, K., Suwanarusk, R., Nosten, F. and Renia, L. (2013). Giemsa-stained wet mount based method for reticulocyte quantification: a viable alternative in resource limited or malaria endemic settings. PLoS One 8(4): e60303.

7. Lee, W. C., Russell, B., Sobota, R. M., Ghaffar, K., Howland, S. W., Wong, Z. X., Maier, A. G., Dorin-Semblat, D., Biswas, S., Gamain, B., Lau, Y. L., Malleret, B., Chu, C., Nosten, F. and Renia, L. (2020). Plasmodium-infected erythrocytes induce secretion of IGFBP7 to form type II rosettes and escape phagocytosis. Elife 9: e51546.

8. Ribacke, U., Moll, K., Albrecht, L., Ahmed Ismail, H., Normark, J., Flaberg, E., Szekely, L., Hultenby, K., Persson, K. E., Egwang, T. G. and Wahlgren, M. (2013). Improved in vitro culture of Plasmodium falciparum permits establishment of clinical isolates with preserved multiplication, invasion and rosetting phenotypes. PLoS One 8(7): e69781. 
9. Sazama, K. (1990). Reports of 355 transfusion-associated deaths: 1976 through 1985. Transfusion 30(7): 583-590.

10. Treutiger, C. J., Carlson, J., Scholander, C. and Wahlgren, M. (1998). The time course of cytoadhesion, immunoglobulin binding, rosette formation, and serum-induced agglutination of Plasmodium falciparum-infected erythrocytes. Am J Trop Med Hyg 59(2): 202-207.

11. Udomsangpetch, R., Wahlin, B., Carlson, J., Berzins, K., Torii, M., Aikawa, M., Perlmann, P. and Wahlgren, M. (1989). Plasmodium falciparum-infected erythrocytes form spontaneous erythrocyte rosettes. J Exp Med 169(5): 1835-1840.

12. Udomsangpetch, R., Webster, H. K., Pattanapanyasat, K., Pitchayangkul, S. and Thaithong, S. (1992). Cytoadherence characteristics of rosette-forming Plasmodium falciparum. Infect Immun 60(11): 4483-4490.

13. Williamson, L. M., Lowe, S., Love, E. M., Cohen, H., Soldan, K., McClelland, D. B., Skacel, P. and Barbara, J. A. (1999). Serious hazards of transfusion (SHOT) initiative: analysis of the first two annual reports. BMJ 319(7201): 16-19.

14. World Health Organization. (2019). World Malaria Report 2019. Switzerland. 